\title{
POTENTIAL OF UAV BASED CONVERGENT PHOTOGRAMMETRY IN MONITORING REGENERATION STANDARDS
}

\author{
Udaya Vepakomma $^{1}$, Denis Cormier ${ }^{1}$, Nelson Thiffault ${ }^{2}$ \\ ${ }^{1}$ FPInnovations, Pointe-Claire, Canada - H9R 3J9 \\ (udayalakshmi.vepakomma; denis.cormier)@fpinnovations.ca \\ ${ }^{2}$ Direction de la recherche forestière, Ministère des Forêts, de la Faune et des Parcs, Québec, Canada - G1P 3W8 \\ Nelson.Thiffault@mffp.gouv.qc.ca
}

KEY WORDS: UAV, point clouds, regeneration, forest management, stocking, space occupancy, height, free-to-grow

\begin{abstract}
:
Several thousand hectares of forest blocks are regenerating after harvest in Canada. Monitoring their performance over different stages of growth is critical in ensuring future productivity and ecological balance. Tools for rapid evaluation can support timely and reliable planning of interventions. Conventional ground surveys or visual image assessments are either time intensive or inaccurate, while alternate operational remote sensing tools are unavailable. In this study, we test the feasibility and strength of UAV-based photogrammetry with an EO camera on a UAV platform in assessing regeneration performance. Specifically we evaluated stocking, spatial density and height distribution of naturally growing (irregularly spaced stems) or planted (regularly spaced stems) conifer regeneration in different phases of growth. Standard photogrammetric workflow was applied on the 785 acquired images for 3D reconstruction of the study sites. The required parameters were derived based on automated single stem detection algorithm developed in-house. Comparing with field survey data, preliminary results hold promise. Future studies are planned to expand the scope to larger areas and different stand conditions.
\end{abstract}

\section{INTRODUTION}

Regeneration performance standards that specify acceptable state of regeneration on areas that have been harvested is used across provinces in Canada. Understanding the success of natural or planted regeneration is critical in ensuring future forest productivity (Solarik et al. 2010, avoid poor forest renewal or habitat for wildlife (Roberts et al. 2006). Periodic monitoring over different stages of regeneration to identify regeneration gaps, understocked areas or seedling failure / mortality is essential for reliably planning interventions (Thiffault et al. 2014, Chabaane et al. 2005). However, regeneration after harvest often does not take place in large areas but rather within smaller blocks, posing an operational challenge for surveillance.

Conventionally, foresters use ground surveys in sampled areas to assess stock or visually interpret high resolution aerial imagery for qualitative assessment of regeneration. These are time intensive or inaccurate. Notable advances have been made to automatically assess characteristics like individual tree detection for stocking (Pouliot \& King 2005) or brush competition (Pouliot et al. 2006) over larger areas using high resolution imagery but these methods have neither been fully tested nor available operationally. Initial research on lidar holds promise, specifically to characterize structure of small vegetation (Martinuzzi et al. 2009, but relatively high costs for obtaining repeat surveys of high density point clouds in small dispersed areas restricts its usage.

Due to limited infrastructure requirements of modern photogrammetric techniques and ease of acquiring convergent images in remote, small and scattered areas like those of regenerating stands, we presume that close-range photography on a UAV platform has potential use for assessing regeneration performance. Hence, our present objective is to test the feasibility and strength of this new technology in evaluating stocking, spatial density distribution and height-class distribution in naturally growing (irregularly spaced stems) or planted (regularly spaced stems) conifer regeneration. In this paper, we present our initial results and recommendations for the future studies.

\section{MATERIAL \& METHODS}

\subsection{Study sites and ground measurements}

\subsubsection{Plantation (evenly spaced stems)}

The Ministère des Forêts, de la Faune et des Parcs du Québec established a network of long-term experimental sites to track success of plantation. We have selected one of these sites, located near the municipality of Saint Donat, about $200 \mathrm{~km}$. northwest of Montreal (QC, Canada). Trees were tagged and are being measured for DBH and height periodically, with the last assessment conducted in 2009. This 2 ha white spruce (Picea glauca (Moench) Voss) plantation was established in 1995 on a fairly flat terrain, of which a block of 0.4 ha is considered for this study. This stand is in a late sapling phase (Figure 1).

In June 2015, total tree height and tree height to the last whorl were measured for 9 randomly selected trees to match the image acquisition in June 2014. . In addition, four plots of size $8 \mathrm{~m}$ $\times 10 \mathrm{~m}$ distributed within a 0.4 ha block were also established. Each plot was sub-divided into $4 \mathrm{~m} \times 5 \mathrm{~m}$ quadrats to count the total number of individuals per species (white spruce or balsam fir, Abies balsamea (L.) Mill.), total height and height up to the last whorl of the tallest tree. Tree heights were measured using a pole. The

*Corresponding author 
historical data of 2009 was used to understand general growth on the site.

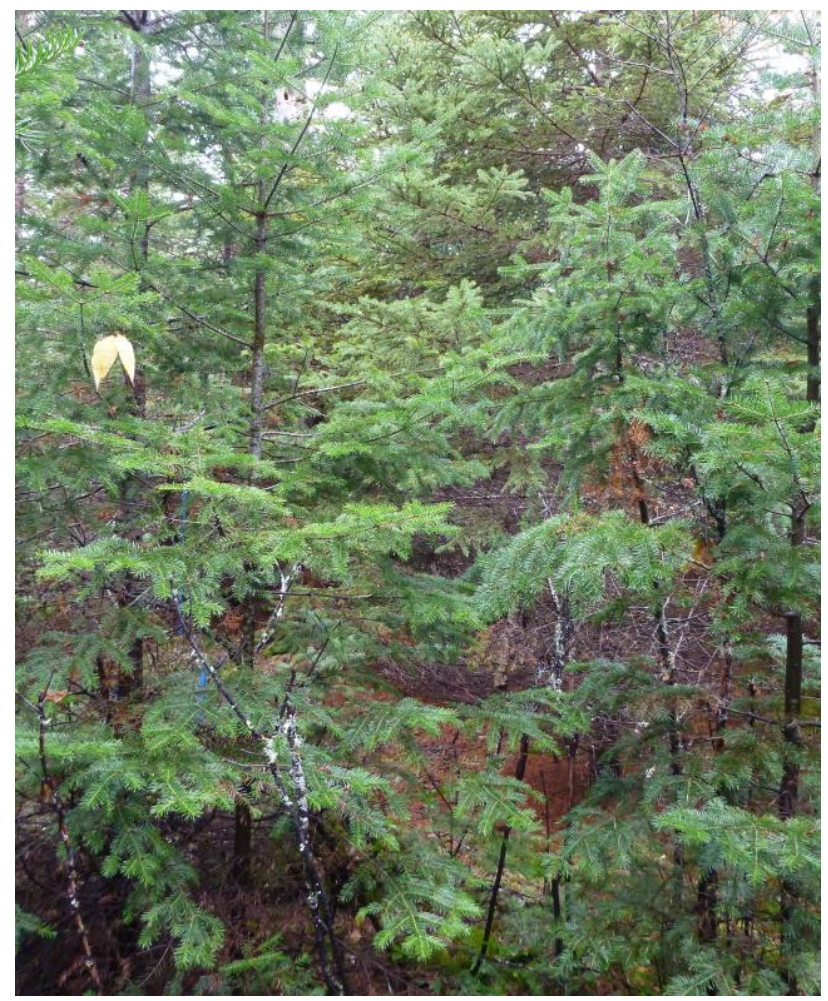

Figure 1. Ground photo showing general structure of dense regeneration of the 20 year old plantation in Saint Donat, Quebec.

\subsubsection{Natural regeneration (irregularly spaced stems)}

This 6 ha. site is part of a recently (2007) harvested block located approximately $100 \mathrm{~km}$. south of the city of Grande Prairie in Alberta, Canada. This block has naturally regenerating loblolly pine (Pinus taeda L.), sometimes mixed with white spruce. The terrain is rolling and highly undulating. This stand is in an early sapling phase (Figure 2). A block of 0.4 ha area is considered for this study.

To assess conifer density, synchronously an independent ground survey was also conducted during the time of UAV image acquisition in 2014 . Three plots of size $4 \mathrm{~m} \times 4 \mathrm{~m}$ respectively representing low, medium and high density areas were established in the test site. The corners of each plot were surveyed with a DGPS (Trimble GeoXH, better than $30 \mathrm{~cm}$ accuracy). Each plot was further sub-divided into $2 \mathrm{~m} \times 2 \mathrm{~m}$ quadrats to count the total number of individuals of pine, spruce and hardwoods (if any), and note the maximum and minimum height of pine individuals. DGPS locations of highest individuals were noted.

\subsection{UAV setup and data acquisition}

The airborne campaigns were conducted in June (Saint Donat) and September (Grande Prairie) of 2014 using a Skyranger quadcopter equipped with an EO camera. Skyranger is a fully

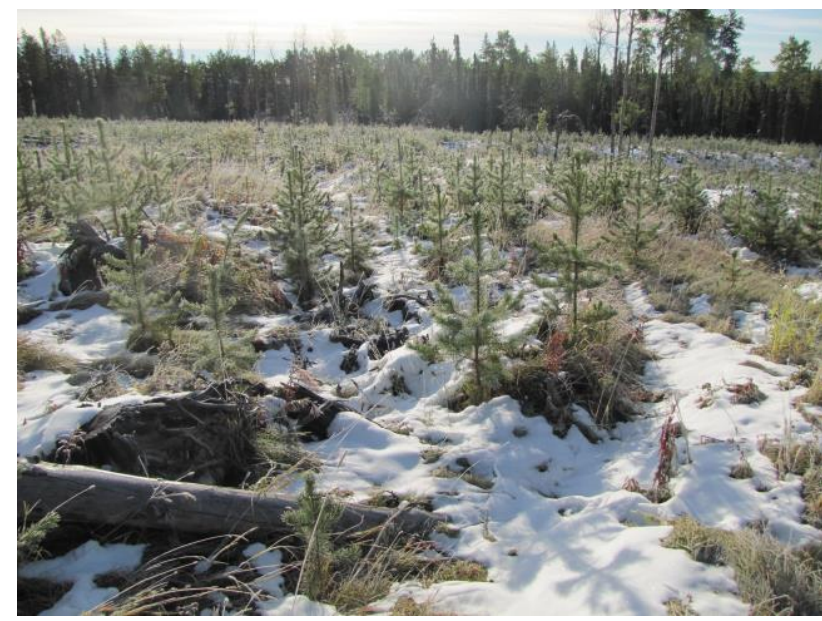

Figure 2. Ground photo showing general structure of the naturally regenerating site near Grande Prairie, Alberta.

assembled $2.4 \mathrm{~kg}$ UAV. It is equipped with an Inertial Navigation System (3-axis gyroscope, 3-axis accelerometer, 3axis magnetometer, GPS receiver, and static pressure sensor) and a sonar sensor for precision AGL altitude measurement.

\begin{tabular}{lcc}
\hline Study area & Plantation & Natural \\
\hline Flight Altitude (m) & 120 & 60 \\
Sidelap (\%) & 75 & 75 \\
Frontlap(\%) & 75 & 75 \\
Viewing angle & Nadir & Nadir \\
Flight speed (km/hr) & 5.9 & 7.3 \\
Capture interval (sec) & 20 & 6 \\
Images acquired & 86 & 699 \\
Images used & 86 & 698 \\
Mosaic area (ha) & 2 & 6 \\
Image format & DNG & JPEG \\
GSD (cm) & 2.2 & 1.1 \\
Total keypoint observations & 40000 & 40000 \\
Total dense 3D points & $10,620,445$ & $52,550,056$ \\
Mean reprojection error (pixel) & 1.05 & 0.86 \\
\# Targets / reference & 6 & 19 \\
Target DGPS accuracy (m) & 0.1 & 0.3 \\
\hline
\end{tabular}

Table 1. Summary of the images acquired at the plantation (Saint Donat, QC) and natural (Grande Prairies, AL) test sites

The EO camera on-board is a SUNEX MT9F002 RGB model with $1.4 \mu \mathrm{m}$ pixel size and $4602 \times 3288$ pixel detector at $13 \mathrm{fps}$. The entire system, as per the manufacturer's description, is expected to be stable and resistant to extreme temperatures and wind gusts.

\subsection{UAV data processing}

A total of 86 and 698 images from the flights conducted over both study sites were used for $3 \mathrm{D}$ reconstruction, dense point 
clouds and finally orthomosaics using Agisoft's Photoscan photogrammetric software. The imagery, synchronised GPS position of each image and DGPS positions of reference targets were used as inputs (Table 1). The processing steps included automatic aero-triangulation, bundle-adjustment, dense 3D point cloud generation and ortho mosaicking (Figure 3).
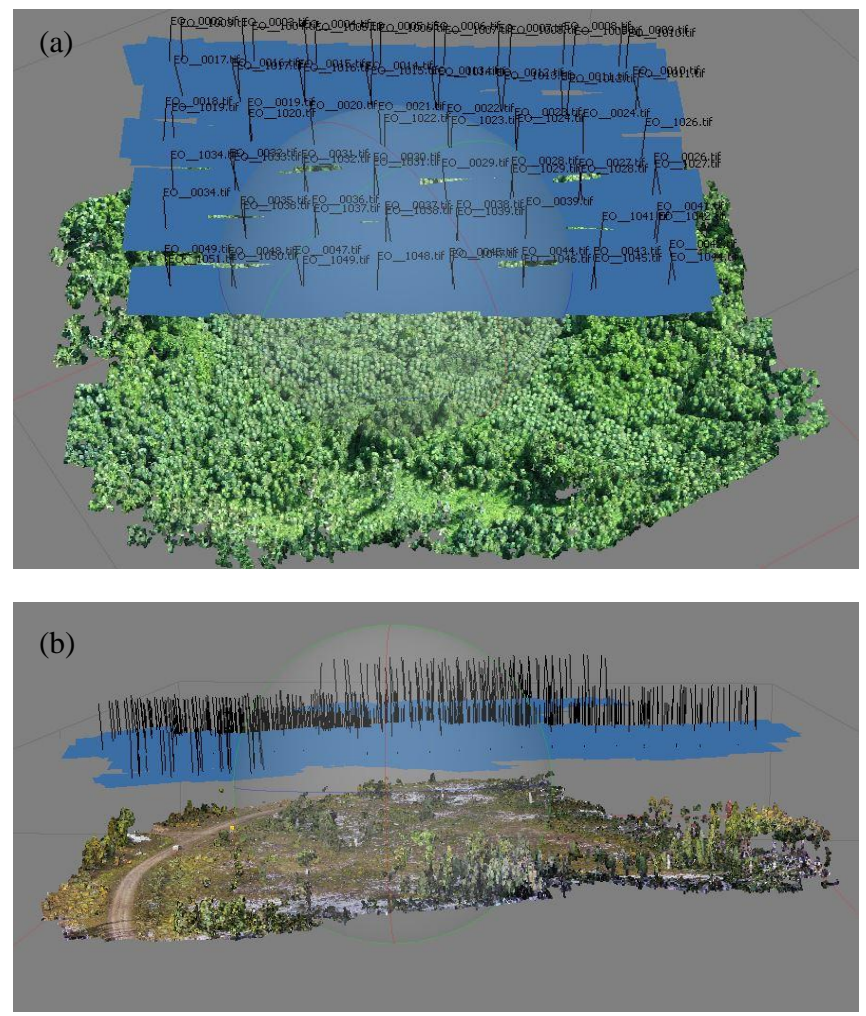

Figure 3. Image acquisition and mosaic of the two test sites. (a) 86 images at $120 \mathrm{~m}$ altitude on evenly spaced plantation near Saint Donat, Quebec (b) 699 images at $60 \mathrm{~m}$ altitude on naturally regenerating block in Grande Prairie, Alberta.

\subsection{Individual stem identification}

Extending the methodology developed for stem identification on lidar surfaces (Vepakomma et al. 2015), the location of individual stem tops was determined by assuming that the pixel associated with the peak of the stem will be higher than its surroundings within a defined buffer on the Digital Surface Model (DSM). DSM was generated using a TIN on the 3D point clouds. DSM was smoothed twice by a Gaussian filter. A local maxima filter with a circular non-overlapping (moving) window of a radius of $35 \mathrm{~cm}$ was applied to the DSM, to determine local peak points. This radius was fixed based on field observation of average crown size in the study sites. Stem height was estimated as difference of the DSM height at the extracted peak from the nearest visible ground.

\subsection{Spatial density and Stocking}

A $2 \mathrm{~m} \times 2 \mathrm{~m}$ grid was created across the study sites and occurrences of a desired type of stem (e.g., pine) were noted within each grid. Total number of identified stems per hectare in a given $2 \mathrm{~m} \times 2 \mathrm{~m}$ cell estimated per hectare is the cell stem density. Spatial density map describes the pattern of stem density on a given site.

Stocking is the growing space required at maturity for each individual stem in a stand. To assess this, a $4 \mathrm{~m}^{2}$ circular area is buffered around each stem. Stocking is estimated as the percentage of the total non-overlapping surface area of this buffer to the total area of the stand.

\section{RESULTS AND DISCUSSION}

\subsection{Plantation}

Figure 4 shows the automated extraction of individual stems in part of the study block. Tree heights estimated by the imagematching method showed an average underestimation of about $0.39 \mathrm{~m}$ when compared to the field measurement of random trees. The correlation of tree heights between the two methods of measurement is over $90 \%$ showing a fairly good level of agreement. Based on the four plots measured on ground, the total tree count of the planted trees in each quadrat matched very well (over 98\%) indicating that the adopted method can reliably discern the single regenerating planted tree locations.
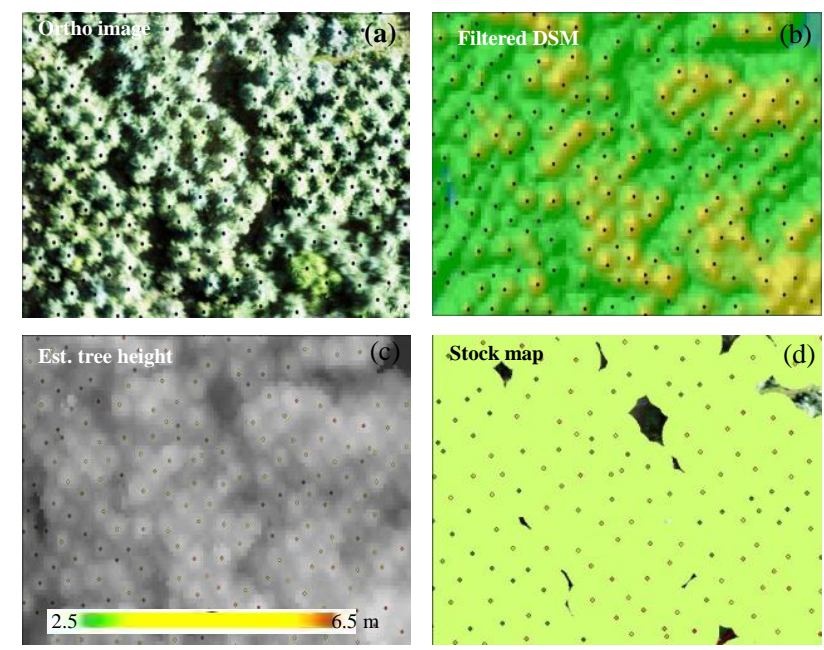

Figure 4. A $20 \mathrm{~m} \times 16 \mathrm{~m}$ sample area of the regenerating plantation. Automated extraction of the individual stems (dots) overlaid on (a) the ortho-image (b) DSM. (c) Shows a grid of the estimated tree height and (d) spatial map of stocking showing regeneration gaps (dark areas) in the evenly spaced plantation (Saint Donat, QC).

In general, compared with the historical field assessment in 2009 in a contiguous area of 0.2 ha block, we observed that the total number of individuals in the block is higher than values obtained from the field assessment (Table 2), and shift in the height-class distribution shows growth over time (Figure 5). Maximum height remained the same. Higher density, lower minimum and average height compared to 2009 indicates potential recruitment in the plantation. The analysis also indicated that $94 \%$ of the area is well stocked on this site (Figure 4d). 


\begin{tabular}{lcc}
\hline Planted & Field & Estimated \\
\hline \# ind. & 160 & 196 \\
Max. Height & 6.9 & 7 \\
Min. Height & 3.1 & 1.67 \\
Average Height & 5.57 & 4.85 \\
\hline
\end{tabular}

Table 2. Summary comparison of estimated (2014) and measured (2009) planted seedling dimensions.

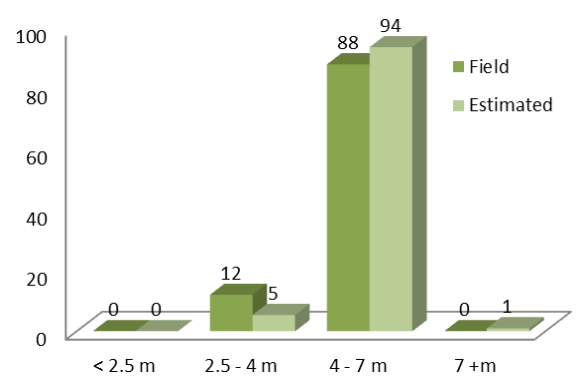

Figure 5. Height- class distribution as per the field (2009) and automatic estimation of the experimental plantation.

\subsection{Natural regeneration}

A sample of the naturally generating site can be seen in Figure 6a. Saplings are irregularly spaced and their size is highly variable (Figure 6b). The numbers of individuals were fairly well identified by the automated extraction of trees algorithm on the 3D reconstructed DSM but stem height were underestimated in the naturally regenerating test plots (Figure 6d). This could be attributed to poor quality of images, low light and unexpected snow fall in the region prior to image acquisition. However, the capability of generating a spatial density as seen in Figure $6 \mathrm{c}$ can help in identifying over-stocked areas that may need tending.

This study in two small experimental sites with completely different conditions (Figures 1 and 2) reveals a proof of concept and understanding of the feasibility of this technology for regeneration assessment. Both set up posed different challenges. Planted areas were uniform and well stocked, while natural regeneration was more scattered and sometimes clustered. While questions remain about the accuracy of tree height estimates in naturally regenerating areas due to poor quality of images, our experience shows that fairly quick acquisition at $120 \mathrm{~m}$ and subsequent analyses of the planted areas in both identifying the individuals and stocking levels is efficient. Between 600,000 and 800,000 ha of forested area is harvested annually in Canada. Because of the legal obligation to ensure proper and sufficient restocking, these areas need to be assessed quickly for regeneration gaps. However, given the scale of observation needed for such assessments, and given the fact that they are usually small, dispersed and in remote locations or sometimes in hazardous site conditions, UAV based convergent photogrammetry could prove to be more efficient in monitoring regeneration standards than conventional ground or alternate remote sensing surveys. Furthermore, current practices are only estimating the stocking level of wanted / targeted species to determine satisfactory regeneration. UAV imagery could also be used at fixed time intervals during stand establishment to evaluate stand growth and adjust AAC calculations.


Figure 6. An example of a sample area of the naturally regenerating site near Grande Prairie, Alberta. (a) A sample plot $(4 \mathrm{~m} \times 4 \mathrm{~m})$ used for field measurements. (b) Shows the 3D rendering of the plot shown in (a). (c) Shows spatial density map (green: $<5000$, orange: 5000-20,000, red: $>20,000$ stems/ha) of plot quadrants in (a). (d) Describes a summary comparison of estimated and measured seedling dimensions.

While UAV based convergent photogrammetry offers a great potential, current regulation from Transport Canada limits its use to within line-of-sight missions without vision enhancing devices. Although this restriction may not pose a problem for small and scattered regenerating stands, it can be restrictive for forestry applications where survey blocks could be very irregular or big, far from a road access or provide limited viewpoints because of surrounding topography and residual stands. However, the regulation agency has indicated being ready to change this rule once "sense and avoid" capability is available to permit safe beyond visual line-of-sight operations.

\section{CONCLUSIONS}

The preliminary results of this study indicate great potential in using close-range photography on a UAV platform for regeneration monitoring, at least in evenly spaced plantations. However, further work is needed in testing its feasibility in naturally growing stands and over larger areas. Another trial is planned for the coming summer (2015) in the same area and we expect improving the methodology. Also, due to lack of field data on precise stem locations and height in a contiguous area, this study could not include the accuracy assessment of the automated single stem algorithm, which will be addressed in future studies. This algorithm was, however, tested to be reliable using lidar CHMs in a different forest. 


\section{ACKNOWLEDGEMENTS}

This work was partly funded by the Industry Sponsored Collaborative project of Alberta Innovation and Advanced Education as well as through integrated research along the entire forest sector value chain at FPInnovations in partnership with Natural Resources Canada. We would also like to acknowledge the assistance provided by Aeryon Labs Inc (Canada) in the initial acquisition of Saint Donat data.

\section{REFERENCES}

Chabaane, Z.H., Bouafif, H., Khaldi, A., Chakroun, H., and R. Caloz., 2005. Télédétection et analyse spatiale de la régénération forestière post-incendie dans le massif de Boukornine au sud de Tunis. Télédétection 5(1-3), pp. 161-181.

Martinuzzi, S., Vierling, L. A., Gould, W. A., Falkowski, M. J., Evans, J. S., Hudak, A. T., and Vierling, K. T., 2009. Mapping snags and understory shrubs for a LiDAR-based assessment of wildlife habitat suitability. Remote Sensing of Environment, 113(12), pp. 2533-2546

Pouliot, D.A., King, D.J., and Pitt, D.G., 2006. Automated assessment of hardwood and shrub competition in regenerating forests using leaf-off airborne imagery. Remote Sensing of Environment, 102(3), pp. 223-236.

Pouliot, D.A., and King, D.J., 2005. Approaches for optimal automated individual tree crown detection in regenerating coniferous forests. Canadian Journal of Remote Sensing, 31(3), pp 255-267.

Roberts, B.A., Simon, N.P.P., and Deering, K.W., 2006. The forests and woodlands of Labrador, Canada: ecology, distribution and future management. Ecological Research, 21, pp. 868-880.

Solarik, K.A., Lieffers, V.J., Volney, W.J.A., Pelletier, R., and Spence, J.R., 2010. Seed tree density, variable retention, and stand composition influence recruitment of white spruce in boreal mixedwood forests. Canadian Journal of Forest Research, 40, pp. 1821-1832.

Thiffault, N., Hébert, F., Charette, L., and Jobidon, R., 2014. Large spruce seedling responses to the interacting effects of vegetation zone, competing vegetation dominance and year of mechanical release. Forestry, 87(1), pp. 153-164.

Vepakomma, U., Kneeshaw, D. and DeGranpré, L., 2015. Edge influence of natural and anthropogenic linear canopy openings on forest structural patterns investigated using lidar (Landscape Ecology - in review) 